\title{
Iterative range estimation in a sloping-bottom shallow-water waveguide using the generalized array invariant
}

\author{
Chomgun $\mathrm{Cho}^{\mathrm{a})}$ and H. C. Song \\ Scripps Institution of Oceanography, La Jolla, California 92093-0238, USA \\ P. Hursky \\ Heat, Light, and Sound Research, Incorporated, San Diego, California 92130, USA
}

S. M. Jesus

University of Algarve, Faro, Portugal

(Received 21 March 2017; revised 8 June 2017; accepted 15 June 2017; published online 6 July 2017)

The array invariant theory was generalized by incorporating the waveguide invariant $\beta$, referred to as the generalized array invariant. In this paper, the generalized array invariant is extended to mildly range-dependent environments with a sloping bottom where the waveguide invariant is variable in range. Assuming knowledge of the bottom slope, the array invariant can be applied iteratively to estimate the source range starting with $\beta=1$ (i.e., range-independent), which converges toward the correct source range by updating $\beta$ at the previously estimated range. The iterative array invariant approach is demonstrated using a short-aperture vertical array $(2.8-\mathrm{m})$ in a sloping-bottom shallow-water waveguide from the Random Array of Drifting Acoustic Receivers 2007 experiment, where a high-frequency source $(2-3.5 \mathrm{kHz})$ close to the surface $(6-\mathrm{m})$ was towed between 0.5 and $5 \mathrm{~km}$ in range with the water depth varying from 80 to $50 \mathrm{~m}$. (ㅇ 2017 Acoustical Society of America. [http://dx.doi.org/10.1121/1.4990670]

[JFL]

Pages: $55-60$

\section{INTRODUCTION}

The array invariant, first proposed by Lee and Makris, ${ }^{1}$ has been successful in achieving robust source-range estimation in shallow-water acoustic environments. ${ }^{2,3}$ The approach is based on the dispersion characteristics in ideal waveguides and involves conventional plane-wave beamforming, exploiting multiple arrivals separated in beam angle and travel time, i.e., beam-time migration. The array invariant $^{1}$ was extended to general waveguides by incorporating the waveguide invariant $\beta$, referred to as the generalized array invariant. ${ }^{3}$ For ideal waveguides and reflectiondominated environments, the waveguide invariant is approximately unity $(\beta \approx 1)$ for small grazing angles (e.g., $<20^{\circ}$ ). As a result, the original array invariant ${ }^{1}$ was assumed to be independent of the waveguide invariant. Using a short- ${ }^{2}$ or long-aperture ${ }^{3}$ vertical array, the generalized array invariant for source-range estimation was demonstrated in shallowwater environments for relatively high-frequency sources (e.g., above $1 \mathrm{kHz}$ ), with minimal knowledge of the environment and computational efficiency.

The inclusion of the waveguide invariant $\beta$ in the formulation $^{2-6}$ implies that the generalized array invariant can be extended to mildly range-dependent environments where mode propagation is adiabatic and thus the waveguide invariant is meaningful via the generalized waveguide invariant. ${ }^{7}$ The objective of this paper is to demonstrate the array invariant-based source-range estimation in shallow-water

\footnotetext{
${ }^{\text {a)} E l e c t r o n i c ~ m a i l: ~ c h o m g u n @ g m a i l . c o m ~}$
}

environments with range-varying bottom bathymetry using at-sea data.

The waveguide invariant for an ideal waveguide with a sloping bottom was derived by D'Spain and Kuperman using the adiabatic approximation, ${ }^{8}$ which is simply a ratio of the water depth at the source location to that at the receiver location. The main drawback for source range estimation is that an accurate value of $\beta$ requires prior knowledge of the water depth at the source range $\left(r_{0}\right)$ to be estimated. Assuming that the bottom slope is known to the receiver, we propose an iterative array invariant to estimate the source range starting with $\beta=1$ (i.e., range-independent), which converges toward the correct source range by updating $\beta(\hat{r})$ at the previously estimated range $(\hat{r})$. The iterative array invariant approach will be experimentally verified using a short-aperture vertical array $(2.8 \mathrm{~m})$ in a sloping-bottom shallow-water waveguide, where a high-frequency source $(2-3.5 \mathrm{kHz})$ near the surface (6-m) was towed between 0.5 and $5 \mathrm{~km}$ in range with the water depth varying from 80 to $50 \mathrm{~m}$.

The paper is organized as follows. Section II reviews the generalized array invariant and generalized waveguide invariant for an ideal waveguide with a sloping bottom, including a high-order approximation that is derived in the Appendix. Then the iterative approach to range estimation is described in conjunction with the array invariant. Section III describes the Random Array of Drifting Acoustic Receivers 2007 (RADAR07) experiment, ${ }^{9}$ conducted off Setúbal, Portugal, in July 2007, with a major focus on the source-tow run in a sloping environment. In Sec. IV, the performance of tracking the towed source using the iterative array invariant is presented, followed by a summary in Sec. V. 


\section{GENERALIZED ARRAY INVARIANT FOR A SLOPING BOTTOM}

The generalized array invariant that includes the waveguide invariant $\beta$ is derived in the literature. ${ }^{3,4}$ The source range $r_{0}$ can be estimated simply from

$$
r_{0}=-\beta\left(\frac{c}{\chi}\right)
$$

where $c$ is the local sound speed used for beamforming. For an ideal waveguide with $\beta=1$, this formula reduces to the original array invariant. ${ }^{1}$

The array invariant parameter $\chi$ is defined as

$$
\chi \equiv \frac{d}{d t}(\cos \theta)=\frac{d}{d t} \sqrt{1-\sin ^{2} \theta}=\frac{d}{d t} \sqrt{1-s^{2}},
$$

where $s=\sin \theta$ with the grazing angle $\theta$ from the horizontal and $t$ is the travel time. The above equation can be expressed as an elliptic curve in the beam-time $(s, t)$ coordinate (see Fig. 3):

$$
\left(\frac{t-t_{0}}{1 / \chi}\right)^{2}+\sin ^{2} \theta=1
$$

where $1 /|\chi|$ is the horizontal semi-major axis and the center of ellipse is at $\left(0, t_{0}\right){ }^{4}$ The parameter $\chi$ can be estimated numerically from the beam-time migration data, e.g., using the least-squares (LS) approach as described in the Appendix of Ref. 3.

\section{A. Generalized waveguide invariant for a sloping bottom}

For an ideal waveguide with a sloping bottom, D'Spain and Kuperman ${ }^{8}$ derived a generalized waveguide invariant such that

$$
\frac{1}{\beta}=\frac{D_{r}}{D_{s}},
$$

where $D_{r}$ and $D_{s}$ are the water depth at the receiver and the source location, respectively. This simple expression was then experimentally verified to analyze the data collected in a bottom-slope shallow-water environment. For a rangeindependent environment, i.e., when $D_{r}=D_{s}$, Eq. (4) reduces to $\beta=1$, which is also valid in many bottom-interacting shallow-water environments.

Since the source range $\left(r_{0}\right)$ is proportional to $\beta$ in Eq. (1), it is important to assess the accuracy of the simple expression Eq. (4). Thus, we have revisited the derivation in the Appendix where Eq. (4) corresponds to a zeroth-order approximation, Eq. (A4). On the other hand, the first-order approximation derived in Eq. (A6), which is almost identical to the analytic expression in Eq. (A1), has an additional term that depends on the grazing angle (or mode number). For an up-slope bottom similar to the RADAR07 environment, the zeroth-order approximation is estimated to be about $6 \%$ larger than the first-order approximation when averaged over up to the bottom critical angle of $\sin 24^{\circ}$ (refer to Fig. 5). Consequently, the trade-off of using the simple expression Eq. (4) with the array invariant would be over-estimation of the source range, as will be confirmed in Sec. IV.

\section{B. Iterative array invariant}

The array invariant-based source-range estimation using Eq. (1) includes $\beta$, which is approximately unity in many shallow-water environments. ${ }^{7}$ For range-dependent sloping bottom, however, an accurate value of $\beta$ in Eq. (4) requires prior knowledge of the water depth $\left(D_{s}\right)$ at the source range $\left(r_{0}\right)$ to be estimated. Assuming the bottom slope is known to the receiver, the array invariant approach can be employed iteratively as follows:

(1) Estimation of the array invariant parameter $\chi$ from the beam-time migration data.

(2) Initial range estimate of $\hat{r}_{0}=-c / \chi$, assuming $\beta_{0}=1$ (i.e., $D_{s}=D_{r}$ ) and $c=1500 \mathrm{~m} / \mathrm{s}$.

(3) Iterative range estimate of $\hat{r}_{k}=\beta_{k} \hat{r}_{0}$ with an updated $\beta_{k}=D\left(\hat{r}_{k-1}\right) / D_{r}$, where $D(x)$ is the water depth at the estimated source range $x$ in the previous iteration.

(4) Convergence check: $\left|\hat{r}_{k}-\hat{r}_{k-1}\right| \leq R_{c}$ (e.g., $100 \mathrm{~m}$ ), a radius of convergence (threshold).

\section{RADAR07 EXPERIMENT}

The RADAR07 experiment was performed on 9-15 July 2007 on the continental shelf off the west coast of Portugal, roughly $23 \mathrm{~km}$ south of Setúbal. ${ }^{9}$ This is a dynamic site with significant internal tide activity due to the complex bathymetric features, including a canyon, seamounts, and a narrow continental shelf. The experiment was a multi-institutional effort between University of Algarve's SiPLAB, Nato Undersea Research Centre (NURC), the Hydrographic Institute (of the Portuguese Navy), Naval Research Laboratory (NRL), and Heat, Light and Sound (HLS) Research, Inc. The experiment was designed to support research in several areas, including matched field tomography and underwater communications. Two research vessels from the Hydrographic Institute of Portugal were used, NRP Don Carlos I and NRP Auriga. Active acoustic signals were transmitted from NRP D. Carlos I towing three different acoustic sources covering $0.5-20 \mathrm{kHz}$ and were received by multiple receive arrays, drifting or moored.

To investigate the proposed iterative array invariant approach, our analysis will focus on the source-tow run carried out on JD 194 (July 13), from 16:38 to 18:19 UTC (about $2 \mathrm{~h}$ ). The schematic of the experiment is illustrated in Fig. 1 along with the bathymetry. A broadband lowfrequency source $(0.5-3.5 \mathrm{kHz})$ was deployed to about $6-\mathrm{m}$ depth and towed mostly at a speed of about 3 knots $(1.4 \mathrm{~m} / \mathrm{s})$ by the NRP D. Carlos I along the specified A-F source-tow track in a sloping environment, first in the up-slope and then down-slope direction. The white circle $(\bigcirc)$ in Fig. 1(b) indicates the location of NURC's SLIVA (SLIm Vertical Array) moored in about 87.5-m water depth, consisting of three nested subsets of hydrophones spaced for various frequency bands of interest. In this paper, we will use a subset of 
(a)

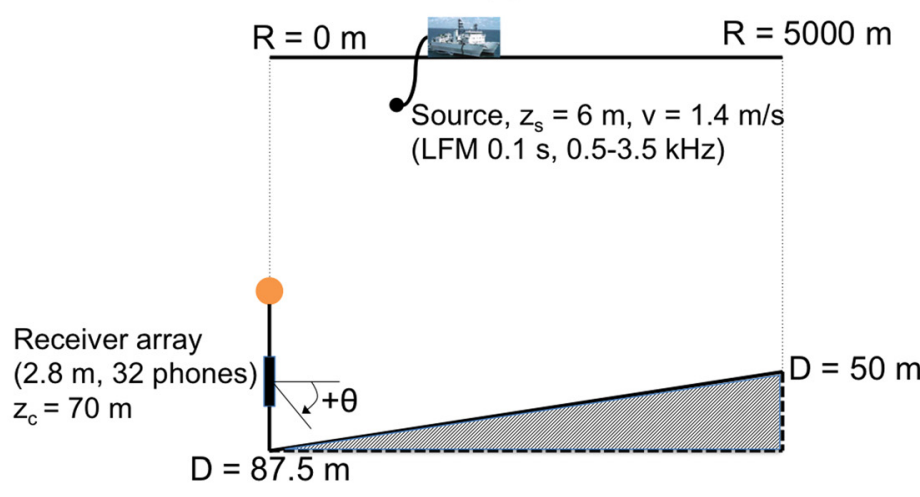

(b)

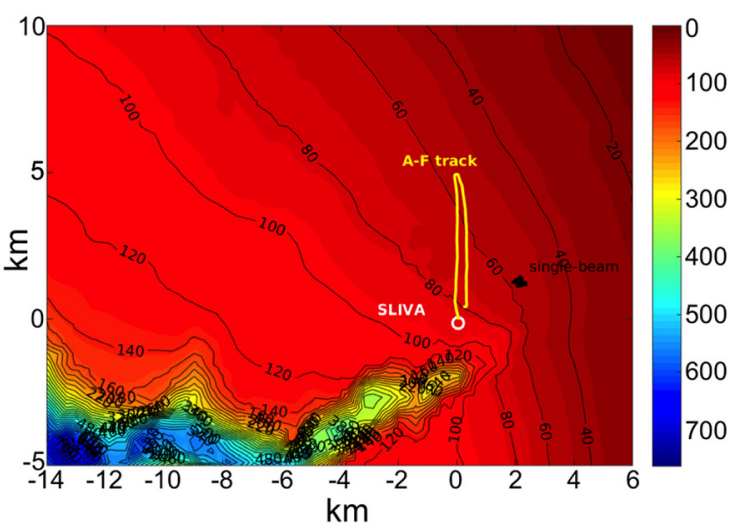

FIG. 1. (Color online) RADAR07 source-tow experiment overview: (a) schematic of the deployed hardware in a simplified sloping environment and (b) complex bottom bathymetry off Setúbal, Portugal, along with the ship track (yellow line), first in the up-slope and then down-slope direction. The open circle ( $\bigcirc$ ) denotes the receiver array location (SLIVA). The water depth is in meters.

32-element line array with a $2.8-\mathrm{m}$ aperture centered around $70-\mathrm{m}$ depth. Note that the bottom bathymetry is approximated by a simple sloping bottom in Fig. 1(a), facilitating the iterative approach based on the knowledge of the slope. This work utilizes $100-\mathrm{ms}, 0.5-3.5 \mathrm{kHz}$ linear frequency modulated (LFM) chirp channel probe transmitted at various ranges $(0.5-5 \mathrm{~km})$ during the source-tow run.

Environmental data included water-column sound-speed profiles (SSPs) using conductivity, temperature, and depth (CTD) and thermistor strings. Two SSPs measured prior to the source-tow run were averaged out in Fig. 2(a), which is a downward-refracting environment with the mixed layer depth down to $10 \mathrm{~m}$. The towed source at $6-\mathrm{m}$ depth $(*)$ is in the mixed layer, while the receiver array $(O)$ is well below the thermocline at $70-\mathrm{m}$ depth. The source near the surface can excite high-order modes that interact with surface and bottom; thus, these modes behave like ones in an ideal waveguide from which the array invariant originated. As a result, all the high-order modes (i.e., ray arrivals) can be used to estimate the source range based on the array invariant.

An example of channel impulse response (CIR) at $0.7 \mathrm{~km}$ range is displayed in Fig. 2(b), indicating six distinct arrivals with a delay spread of about $80 \mathrm{~ms}$. The plot is normalized by the maximum intensity and displayed in $\mathrm{dB}$. Few hydrophones (e.g., at 68.6 and $69.5 \mathrm{~m}$ ) were not working properly; thus, they are excluded for plane-wave beamforming.

\section{SOURCE TRACKING IN A SLOPING ENVIRONMENT}

The RADAR07 data collected during the source-tow run (16:38-18:19 UTC) are analyzed to track the source traveling in a sloping environment as depicted in Fig. 1. The source signal was a $100-\mathrm{ms}, 0.5-3.5 \mathrm{kHz}$, LFM chirp transmitted at 0.2 -s intervals for $4 \mathrm{~s}$ (i.e., 20 chirp transmissions) and then repeatedly every $30 \mathrm{~s}$ at various ranges from $0.5 \mathrm{up}$ to $5 \mathrm{~km}$. To improve the beam resolution given the aperture of the array $(2.8 \mathrm{~m})$, only the upper-half frequency band (i.e., $50-\mathrm{ms}, 2-3.5 \mathrm{kHz})$ is utilized for matched-filtering and beamforming.

\section{A. Beam-time migration}

The beam-time migration $(s, t)$ is presented in Fig. 3 for three representative source ranges: (a) $0.7 \mathrm{~km}$, (b) $2.8 \mathrm{~km}$, and (c) $4.9 \mathrm{~km}$. The water depth at the corresponding range (a)

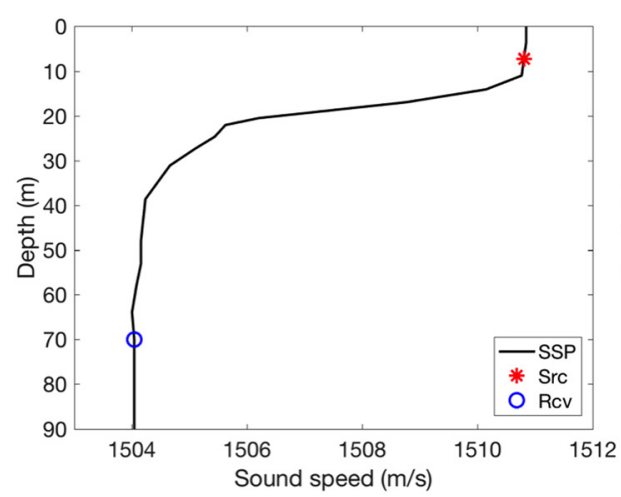

(b)

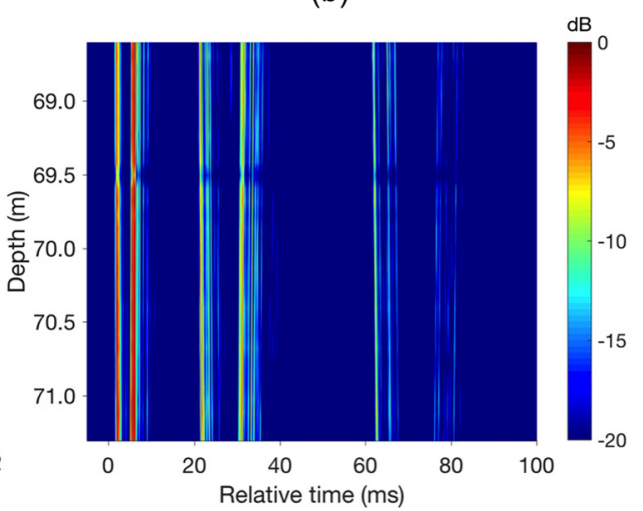

FIG. 2. (Color online) (a) A representative sound speed profile prior to the source-tow run on JD 194. The source at 6 -m depth (*) is in the surface mixed layer, while the receiver at $70-\mathrm{m}$ depth $(\bigcirc)$ is well below the thermocline in a downward-refracting environment. (b) Channel impulse response (CIR) measured at a range of $0.7 \mathrm{~km}$, indicating a delay spread of about $80 \mathrm{~ms}$ with 6 distinct arrivals. The plot is normalized by the maximum intensity and displayed in dB. Few hydrophones (e.g., at 68.6 and $69.5 \mathrm{~m}$ ) were not working properly; thus, they are excluded for conventional plane-wave beamforming. 
(a)

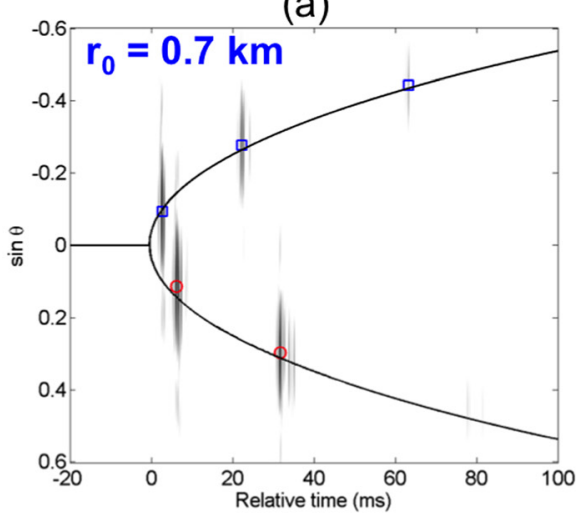

(c)

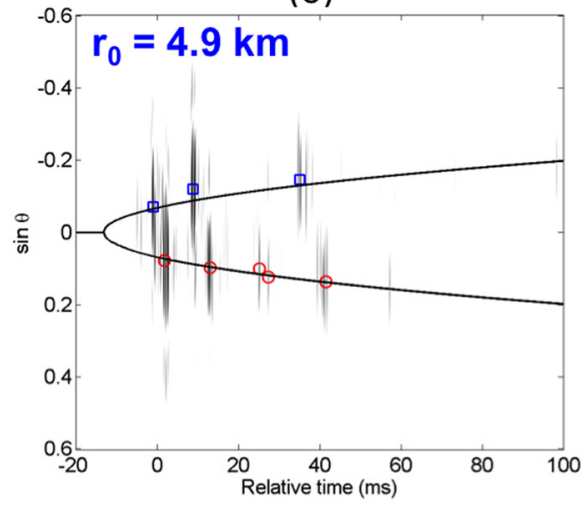

(b)

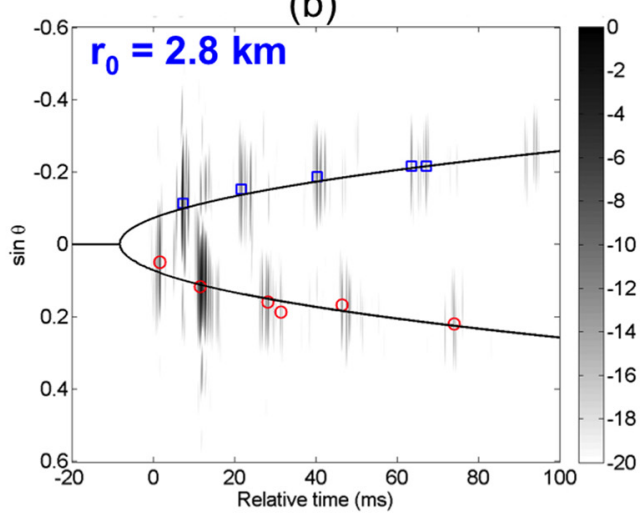

(d)

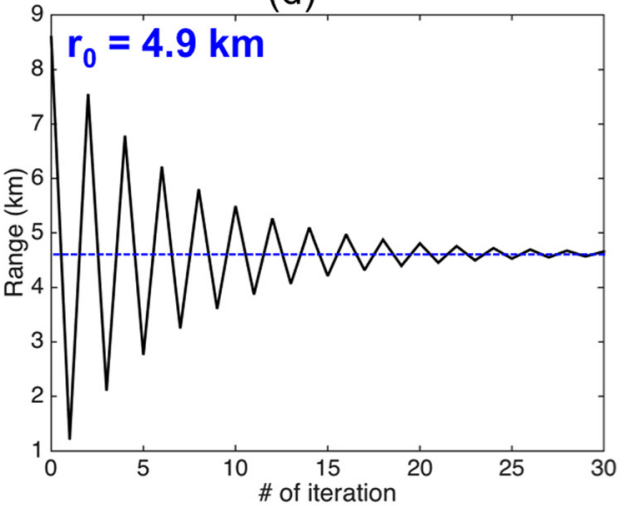

FIG. 3. (Color online) Beam-time migration at three representative ranges: (a) $0.7 \mathrm{~km}$ (16:41), (b) $2.8 \mathrm{~km}$ (17:06), and (c) $4.9 \mathrm{~km}$ (17:29 UTC). The water depth at the corresponding range is 75 , 62 , and $55 \mathrm{~m}$. A positive beam angle represents an up-going path (red circles), and the dynamic range is $20 \mathrm{~dB}$. Note that the curvature of the elliptic curve increases with range. A different number of distinct arrivals (circles and squares) is used to estimate the array invariant parameter $\chi$ at different ranges. (d) Iterative range estimation in a sloping environment for a source at $4.9 \mathrm{~km}$ range corresponding to (c). The range estimate is initially $8.6 \mathrm{~km}$ assuming $\beta=1$ (range-independent) and $c=1500 \mathrm{~m} / \mathrm{s}$, but gradually converges toward $4.6 \mathrm{~km}$ in a zig-zag fashion with a relative range error of $-6 \%$. The performance of iterative range estimation is summarized in Table I. is 75,62 , and $55 \mathrm{~m}$, respectively. The vertical axis denotes the beam $(s=\sin \theta)$ with a positive angle $\theta$ defined for an up-going paths (red circles), and the horizontal axis is the relative travel time $(t)$. A varying number of arrivals were identified at different ranges, determined by a threshold set to find peaks (e.g., $-10 \mathrm{~dB}){ }^{3}$ For instance, there are two upgoing (circles) and three down-going (squares) arrivals at $0.7 \mathrm{~km}$, whereas many more arrivals up to 11 are captured at $2.8 \mathrm{~km}$.

Following the steps in Sec. II B, an elliptic curve (solid line) that best fits the identified arrivals (circles and squares) in the LS sense $^{3}$ can be drawn with an appropriate array invariant parameter $\chi$ (refer to Table I). It is noticeable that the curvature of the ellipses increases as the range increases. The iterative array invariant approach to range estimation is illustrated in Fig. 3(d) for the source at $4.9 \mathrm{~km}$ range. The range estimate is initially $8.6 \mathrm{~km}$ assuming $\beta=1$ and $c=1500 \mathrm{~m} / \mathrm{s}$, but gradually converges over the iteration toward $4.6 \mathrm{~km}$ in a zig-zag fashion, with a relative range

TABLE I. Iterative source-range estimation from beam-time migration shown in Fig. 3 at various ranges.

\begin{tabular}{lcccccc}
\hline \hline $\begin{array}{l}\text { Source } \\
\text { range } \\
\left(r_{0}\right)\end{array}$ & $\begin{array}{c}\text { Water depth } \\
\left(D_{s}\right)\end{array}$ & $\begin{array}{c}\beta\left(r_{0}\right) \\
=D_{s} / D_{r}\end{array}$ & $\chi$ & $\begin{array}{c}\text { Initial estimate } \\
\text { with } \beta=1\end{array}$ & $\begin{array}{c}\text { Range } \\
\left(\hat{r}_{0}\right)\end{array}$ & $\begin{array}{c}\text { Relative } \\
\text { range } \\
\text { error }\end{array}$ \\
\hline $\begin{array}{l}\text { (a) } 0.7 \mathrm{~km} \\
\text { (b) } 2.8 \mathrm{~km}\end{array}$ & $75 \mathrm{~m}$ & 0.86 & -1.60 & $0.9 \mathrm{~km}$ & $0.9 \mathrm{~km}$ & $25 \%$ \\
(c) $4.9 \mathrm{~km}$ & $55 \mathrm{~m}$ & 0.71 & -0.31 & $4.8 \mathrm{~km}$ & $3.3 \mathrm{~km}$ & $17 \%$ \\
\hline \hline
\end{tabular}

error of $-6 \%$. The performance of iterative range estimation for the above three ranges is summarized in Table I.

\section{B. Source-range tracking}

During the source-tow run, the LFM chirp signal was transmitted continuously at $0.2-\mathrm{s}$ intervals for $4 \mathrm{~s}$ (i.e., 20 chirp transmissions) and then repeated every $30 \mathrm{~s}$ over almost $2 \mathrm{~h}$. Thus, for each range, the mean range estimate was obtained from 20 transmissions. The overall performance of range estimate is presented in Fig. 4 over the entire source-tow run. The solid line is based on the ship GPS data where the towed source was assumed to be about $70 \mathrm{~m}$ behind the actual GPS location, although the offset is relevant only at close ranges (e.g., $<1 \mathrm{~km})$.

The open circles $(\bigcirc)$ obtained with $\beta=1$ do not take into account the variability of $\beta(r)$ in a sloping rangedependent environment. As a result, the range error increases significantly with range such that the relative range error exceeds $100 \%$ at about $5 \mathrm{~km}$ range. On the other hand, the iterative array invariant approach $(\bigcirc)$ even with a simplified sloping bottom in Fig. 1(a) provides good performance over the entire source track with the relative range error of about $18 \%$. As described in the Appendix, the source range is mostly over-predicted because the simple expression for the waveguide invariant in Eq. (4) is always larger than the exact value. A few exceptions of under-prediction at around $5 \mathrm{~km}$ (e.g., $-6 \%$ ) are likely due to the mismatch between the simplified sloping bottom and the actual bottom bathymetry. Additional uncertainty is attributed to the fact that the derivation of $\beta$ is based on a two-dimensional sloping bottom, 
(a)

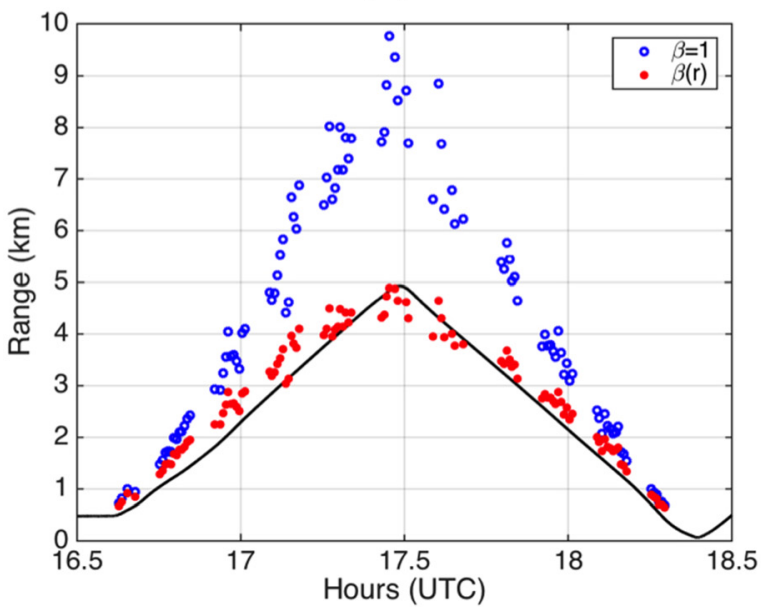

(b)

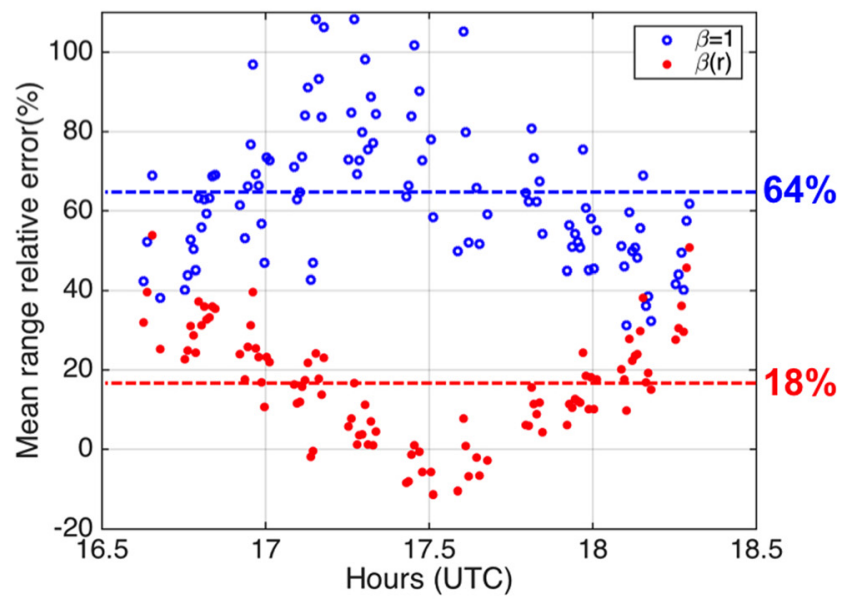

FIG. 4. (Color online) (a) Source-range estimation during the source-tow run for about $2 \mathrm{~h}(16: 38-18: 19)$ : assuming $\beta=1$ (open circles, blue) and using the iterative approach with $\beta(r)$ (solid circles, red). The solid line is based on the ship GPS. (b) Corresponding relative range errors (\%). The mean relative range error with the iterative approach is about $18 \%$.

neglecting the three-dimensional propagation effect in the RADAR07 environment.

\section{SUMMARY}

The generalized array invariant that includes the waveguide invariant $\beta$ has been successful for source range estimation with minimal environmental information and using a short-aperture vertical array in shallow water. In this paper, the array invariant was extended to a range-dependent environment with a sloping bottom where $\beta$ requires prior knowledge of the water depth at the source range to be estimated. To get around the problem, an iterative approach was proposed, which starts with $\beta=1$ but gradually converges toward the correct source range by updating $\beta$ at the previously estimated source range. The iterative array invariant approach was demonstrated using a short-aperture vertical array $(2.8 \mathrm{~m})$ in a sloping-bottom shallow-water environment, where a high-frequency source $(2-3.5 \mathrm{kHz})$ near the surface (6-m) was towed between 0.5 and $5 \mathrm{~km}$ in range with the water depth varying from 80 to $50 \mathrm{~m}$. The mean absolute relative range error was about $18 \%$ during the entire sourcetow run.

\section{ACKNOWLEDGMENT}

C.C. and H.C.S. were supported by the Office of Naval Research under Grant No. N00014-13-1-0510. The authors gratefully acknowledge the captains and crew of NRP Don Carlos I and NRP Auriga from the Portuguese Navy, the engineering staff from NURC, and the graduate students from the University of Algarve, who helped perform the RADAR07 experiment. The participation of HLS Research Inc. in the RADAR07 experiment was funded through a Phase 2 STTR, Topic N05-T022, titled Acoustic Communications Modem Using MIMO Technology, performed for Bob Headrick and the ONR Ocean Acoustics program.

\section{APPENDIX: WAVEGUIDE INVARIANT FOR AN IDEAL WAVEGUIDE WITH A SLOPING BOTTOM}

The starting point is the expression for the waveguide invariant $\beta\left(r_{0}\right)$ derived in Ref. 8 for an ideal waveguide with range-varying bottom bathymetry where mode propagation is adiabatic [refer to Eq. (22) of Ref. 8]:

$$
\frac{1}{\beta\left(r_{0}\right)}=\frac{\left(1-\gamma_{n}^{2}(0) / k^{2}\right)^{1 / 2}}{\gamma_{n}^{2}(0)} \frac{1}{r_{0}} \int_{0}^{r_{0}} \frac{\gamma_{n}^{2}(x)}{\left(1-\gamma_{n}^{2}(x) / k^{2}\right)^{3 / 2}} d x
$$

where $\gamma_{n}(x)$ is the $n$ th-mode vertical wave number, $k$ is the medium wave number, and $r_{0}$ is the source range from the receiver at $r=0$. For the low-order modes where $\gamma_{n}^{2} / k^{2} \ll 1$, we can apply the first-order Taylor approximation ${ }^{10}$ to each of the two power functions containing $\gamma_{n}^{2} / k^{2}$, and Eq. (A1) thus becomes

$$
\frac{1}{\beta\left(r_{0}\right)} \approx\left(\frac{1}{\gamma_{n}^{2}(0)}-\frac{1}{2 k^{2}}\right) \frac{1}{r_{0}} \int_{0}^{r_{0}}\left(\gamma_{n}^{2}(x)+\frac{3 \gamma_{n}^{4}(x)}{2 k^{2}}\right) d x .
$$

Neglecting the product of the two first-order terms (i.e., $\left.1 / 2 k^{2} \times \int_{0}^{r_{0}}\left\{\left[3 \gamma_{n}^{4}(x)\right] / 2 k^{2}\right\} d x\right)$, we obtain

$$
\begin{aligned}
\frac{1}{\beta\left(r_{0}\right)} \approx & \frac{1}{\gamma_{n}^{2}(0)} \frac{1}{r_{0}} \int_{0}^{r_{0}} \gamma_{n}^{2}(x) d x+\frac{1}{\gamma_{n}^{2}(0)} \\
& \times \frac{1}{r_{0}} \int_{0}^{r_{0}} \frac{3 \gamma_{n}^{4}(x)}{2 k^{2}} d x-\frac{1}{2 k^{2}} \frac{1}{r_{0}} \int_{0}^{r_{0}} \gamma_{n}^{2}(x) d x .
\end{aligned}
$$

The first term on the right-hand side, corresponding to a zeroth-order approximation, can be simplified for an ideal waveguide with a sloping bottom:

$$
\frac{1}{\gamma_{n}^{2}(0)} \frac{1}{r_{0}} \int_{0}^{r_{0}} \gamma_{n}^{2}(x) d x=\frac{D_{r}^{2}}{r_{0}} \int_{0}^{r_{0}} \frac{1}{d_{n}^{2}(x)} d x=\left(\frac{D_{r}}{D_{s}}\right),
$$


where $\quad \gamma_{n}(x)=n \pi / d(x), \gamma_{n}(0)=n \pi / d(0), d(x)=D_{r}+A x$ with $A$ representing the bottom slope. The water depths at the receiver and the source locations are $D_{r}$ and $D_{s}$ $=D_{r}+A r_{0}$, respectively. D'Spain and Kuperman ${ }^{8}$ used this formula for the analysis of data collected in a sloping shallow-water environment. For a range-independent case (i.e., $D_{r}=D_{s}$ ), the zeroth-order waveguide invariant becomes equal to one $(\beta=1)$.

The second and third terms on the right-hand side of Eq. (A3), corresponding to the first-order approximation, can be combined into a simple expression

$$
\begin{aligned}
& \frac{1}{\gamma_{n}^{2}(0)} \frac{1}{r_{0}} \int_{0}^{r_{0}} \frac{3 \gamma_{n}^{4}(x)}{2 k^{2}} d x-\frac{1}{2 k^{2}} \frac{1}{r_{0}} \int_{0}^{r_{0}} \gamma_{n}^{2}(x) d x \\
& =\frac{3 n^{2} \pi^{2} D_{r}^{2}}{2 k^{2}} \frac{1}{r_{0}} \int_{0}^{r_{0}} \frac{1}{d^{4}(x)} d x-\frac{n^{2} \pi^{2}}{2 k^{2}} \frac{1}{r_{0}} \int_{0}^{r_{0}} \frac{1}{d^{2}(x)} d x \\
& \left.=\frac{n^{2} \pi^{2} D_{r}^{2}}{D_{r}^{2} k^{2}} \frac{D_{r}+D_{s}}{2 D_{s}}\right)=\sin ^{2} \theta_{n}\left(\frac{D_{r}}{D_{s}}\right)^{2}\left(\frac{D_{\mathrm{avg}}}{D_{s}}\right),
\end{aligned}
$$

where $D_{\text {avg }}=\left(D_{r}+D_{s}\right) / 2$ is the averaged water depth and $\theta_{n}$ is the grazing angle of $n$th mode at the receiver. From Eqs. (A4) and (A5), we obtain an expression for the waveguide invariant $\beta\left(r_{0}\right)$ in an ideal waveguide with a sloping bottom that includes up to the first-order terms:

$$
\frac{1}{\beta\left(r_{0}\right)} \approx\left(\frac{D_{r}}{D_{s}}\right)+\left(\frac{D_{\mathrm{avg}}}{D_{s}}\right)\left(\frac{D_{r}}{D_{s}}\right)^{2} \sin ^{2} \theta_{n}
$$

Besides the water depth at the source and receiver locations, the first-order waveguide invariant $\beta\left(r_{0}\right)$ depends on the grazing angle $\theta_{n}$ at the receiver. For a range-independent case $\left(D_{r}=D_{s}\right)$, the above expression reduces to $\beta=1 /$ $\left(1+\sin ^{2} \theta_{n}\right) \approx \cos ^{2} \theta_{n}$ for small angles (e.g., $\sin \theta<0.4$ ), which is the analytic expression for the waveguide invariant in ideal waveguide suppressing the modal index $(\beta$ $\left.=\cos ^{2} \theta\right) \cdot{ }^{7}$ It should be mentioned that Burenkov ${ }^{11}$ also provided a similar first-order approximation where the factor $\left(D_{\text {avg }} / D_{s}\right)$ was missing in the second term.

To investigate the accuracy of the above approximations, the waveguide invariant $\beta\left(r_{0}\right)$ is evaluated at $2 \mathrm{~km}$ range for an upslope bottom similar to the RADAR07 and is displayed in Fig. 5 as a function of grazing angle (or mode number). The water depths at the receiver and source location are 87.5 and $72.5 \mathrm{~m}$, respectively. While the first-order approximation (dashed line) of Eq. (A6) gradually decreases with an increase in the grazing angle (i.e., high-order modes), it is almost identical to the exact solution of Eq. (A1) (solid line) up to $\sin \theta=0.2$ and the deviation at $\sin \theta$ $=0.5$ is less than $10 \%$. On the other hand, the zeroth-order approximation of Eq. (A4) (dotted horizontal line) has a constant value of $\beta=0.83$, which is about $24 \%$ higher than the first-order approximation at $\sin \theta=0.5$. Although not shown here, similar results were obtained at other ranges. The bottom critical angle for the RADAR07 environment was about $\sin 24^{\circ}=0.4$.

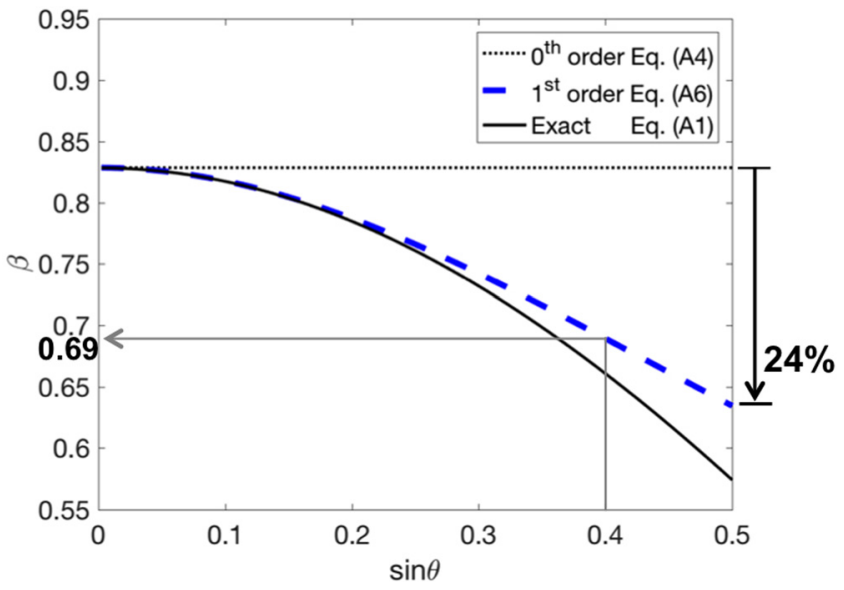

FIG. 5. (Color online) The waveguide invariant $\beta\left(r_{0}\right)$ evaluated at $2-\mathrm{km}$ range for an upslope bottom as a function of grazing angle (or mode number). The water depths at the receiver and source location are 87.5 and $72.5 \mathrm{~m}$, respectively, and the bottom critical angle for the RADAR07 environment is about $\sin \theta=0.4$. The zeroth-order approximation (dotted horizontal line) is $24 \%$ higher than the first-order approximation (dashed blue line) at $\sin \theta=0.5$.

The source-range estimation based on the generalized array invariant is directly affected by the accuracy of $\beta$ via Eq. (1). The average value of $\beta$ from the horizontal up to the critical angle is about 0.78 , which is $6 \%$ less than the zerothorder approximation of 0.83 . Thus, the range estimate based on the generalized array invariant using $\beta=0.83$ is larger than the actual source range. This is evident in Fig. 4 where the majority of the data indicate an over-prediction in source-range estimates with a few exceptions at around the $5 \mathrm{~km}$ range.

${ }^{1}$ S. Lee and N. C. Makris, "The array invariant," J. Acoust. Soc. Am. 119, 336-351 (2006).

${ }^{2}$ C. Cho, H. C. Song, and W. S. Hodgkiss, "Robust source-range estimation using the array/waveguide invariant and a vertical array," J. Acoust. Soc. Am. 139, 63-69 (2016).

${ }^{3}$ H. C. Song and C. Cho, "Array invariant-based source localization in shallow water using a sparse vertical array," J. Acoust. Soc. Am. 141, 183-188 (2017).

${ }^{4}$ H. C. Song and C. Cho, "The relation between the waveguide invariant and array invariant (L)," J. Acoust. Soc. Am. 138, 899-903 (2015).

${ }^{5}$ C. Cho and H. C. Song, "Impact of array tilt on source-range estimation in shallow water using the array invariant," J. Acoust. Soc. Am. 141, 2849-2856 (2017).

${ }^{6}$ H. C. Song, C. Cho, G. Byun, and J. S. Kim, "Cascade of blind deconvolution and array invariant for robust source-range estimation (L), " J. Acoust. Soc. Am. 141, 3270-3273 (2017).

${ }^{7}$ F. B. Jensen, W. A. Kuperman, M. B. Porter, and H. Schmidt, Computational Ocean Acoustics (Springer, New York, 2011), Chap. 2, pp. 133-139.

${ }^{8}$ G. L. D'Spain and W. A. Kuperman, "Application of waveguide invariants to analysis of spectrograms from shallow water environments that vary in range and azimuth," J. Acoust. Soc. Am. 106, 2454-2468 (1999).

${ }^{9}$ C. Soares, S. M. Jesus, P. Hursky, T. Folegot, C. Martins, F. Zabel, L. Quaresma, D.-S. Ko, and E. F. Coelho, "Random Array of Drifting Acoustic Receivers (RADAR'07)" available at http://sapientia.ualg.pt/ handle/10400.1/5277/ (Last viewed 6/28/2017).

${ }^{10}$ K. F. Riley, M. P. Hobson, and S. J. Bence, Mathematical Methods for Physics and Engineering: A Comprehensive Guide (Cambridge University Press, London, 2006), Chap. 4, pp. 139-140.

${ }^{11} \mathrm{~S}$. V. Burenkov, "Distinctive features of the interference structure of a sound field in a two-dimensionally inhomogeneous waveguide," Sov. Phys. Acoust. 35, 465-467 (1989). 